\title{
Physical, cognitive, behavior, and negative emotion in older adults living in China: a national population-based survey
}

This article was published in the following Dove Press journal: Psychology Research and Behavior Management

\section{Yuting Zhang \\ Xiaodong Tan \\ Guanglin Si \\ Yuwei Huang}

Department of Occupational and Environmental Health, School of Health Sciences, Wuhan University, Wuhan 43007I, People's Republic of China
Correspondence: Xiaodong Tan School of Health Sciences, Wuhan University, No. II5, Donghu Road, Wuchang District, Wuhan City, Hubei Province 43007I, People's Republic of China

Tel +860276875859l

Email xiaodongtan20I7@I63.com
Background: Previous studies have concentrated on predictors of exceptional longevity, however, relevant studies have rarely extended to other fields of functioning. To date, little is known about what contributes to the experience of negative emotion in a very old population.

Objective: We aimed to provide a comprehensive key domain of functioning, including physical, cognitive, behavior, and negative emotion in older adults (OAs), followed by determination of predictors of negative emotion.

Methods: Data were collected from a nationally representative sample of 2570 Chinese OAs (aged 60 and above) from the 2014 wave of the Chinese Longitudinal Healthy Longevity Survey. Physical function was assessed using activities of daily living (ADL) and instrumental activities of daily living (IADL) measures. Cognitive function was assessed by the Modified Mini-Mental State Examination (mMMSE). Participants' behavior included smoking, drinking, exercise, physical labor, and social activities. Negative emotion was assessed using four items on affective experiences to create an index of emotional well-being. The associations between various covariates and multiple health outcomes were examined using Pearson's correlation. Multiple linear regressions were established to verify significant variables associated with respondents' negative emotion.

Results: The mean age was 80.20 years $(\mathrm{SD}=8.60)$. The mean ADL total score of the sample was $17.78(\mathrm{SD}=0.89)$, mean IADL total score was $21.93(\mathrm{SD}=3.69)$, the mean mMMSE total score was $21.63(\mathrm{SD}=2.23)$, the mean negative emotion total score was $8.89(\mathrm{SD}=2.64)$. Multiple linear regression analyses revealed that negative emotion was associated with selfperceived low economic status, poor self-rated health condition, poor sleep quality, low IADL function, less regular exercising, living in rural, and being female.

Conclusion: Limited physical functioning and social resources in rural residence may restrict the outpouring of emotion in OAs. Nevertheless, further intensive studies are warranted to provide guidance for the development of better living environment for this elderly population.

Keywords: older adults, physical function, cognition, behavior, negative emotion

\section{Introduction}

With a low fertility rate and rising life expectancy, the number of older adults (OAs) has dramatically increased since the middle of the last century all over the world. ${ }^{1}$ People's Republic of China is also an aging country, with 200 million people aged 60 or above in 2013, accounting for approximately 15\% of People's Republic of China's population. ${ }^{2}$ This aging number has been estimated to rise to 483 million by 
$2050 .^{2}$ In parallel with this rapid aging, the People's Republic of China government has implemented various services and programs to cope with the anticipated needs of the aging population, as well as to improve their quality of life. ${ }^{3,4}$

Despite the great efforts of People's Republic of China government, the mental health needs of Chinese OAs, which are directly associated with their quality of life, have not been addressed adequately. A study conducted by K. W. BOEY reported that sleep disturbance, paranoid ideation, and anxiety were the three most common problems disturbing the elderly in urban People's Republic of China. ${ }^{5}$ Bao-Liang Zhong has addressed that both transient and chronic loneliness are significant predictors of cognitive decline among OAs, both of which have negative effects on the brain health of OAs. ${ }^{6}$ In addition, negative emotion, including depression and stress, may lead to attemptsuicide among OAs. Zhang revealed that anxiety, depressive, sad, and fear symptoms are significantly and independently associated with a higher risk of suicidal ideation among elderly. ${ }^{7}$ The suicide rate among OAs was 34.5 per 100,000 in 2013-14, which has become a serious social problem in People's Republic of China. ${ }^{8}$

Several studies have revealed that health issues, loneliness, isolation, lack of social support, financial problems, and loss of social status after retirement, which lead to mental health issues, are commonly experienced among OAs in Korea. ${ }^{9,10}$ As noted by Moon, risk factors for depression system include lower educational status, lower health status, cognitive impairment, lack of social support, and being female. ${ }^{11}$ Although Chinese OAs suffer from high rates of depression and are faced with the high suicide rate across age groups, however, most of the available studies have only concentrated on predictors of exceptional longevity. Limited studies have ever described to what extent physical health, cognition, and behavior play roles on negative symptoms in People's Republic of China.

To date, studies on predictors of exceptional longevity offer limited information on OAs' behaviors, which may contribute to longevity. Behaviors play essential role throughout the whole life time, especially in elder age due to a sharply increased prevalence of age-related illness. Previous studies have suggested that healthy behaviors, such as frequent exercise and social participation could result in positive perceptions of aging, by contrast, no participation may cause increased feelings of uselessness, loneliness, and anxiety. ${ }^{12,13}$ Other behaviors, such as smoking and alcohol consumption which have been considered to be a part of the culture in People's Republic of China have been established for thousands of years. A British study indicated that those OAs consuming alcohol were more likely to have a fairly sociable lifestyle and a better perceived health condition. ${ }^{14}$ However, there are limited studies concerning the relationships between alcohol consumption and health status, life satisfaction as well as negative emotion in People's Republic of China. ${ }^{15}$

The existing literature on mental health is also lacking due to small datasets and a narrow range of age group. Only few studies have identified whether OAs experience their lives as optimism, happiness or satisfying despite of age-related health restrictions. ${ }^{16}$ A Netherlands population-based observational study found that physical health was hardly related to older people's life satisfaction, whereas poor mental health was strongly relevant for lower life satisfaction. ${ }^{17}$ Meanwhile, Jopp et al found various centenarians reported depressive symptoms. ${ }^{16}$

To extend existing research in healthy longevity, the present study was designed to gain a comprehensive picture of central areas of functioning among OAs. The first aim of the present study was to explore the extent to which physical health, cognitive, behavior, and negative emotion relate to each other. The second aim was to examine which demographic, physical, cognitive, and behavior variables best explain inter-individual differences in negative emotion indicators.

\section{Methods}

\section{Ethical statement}

All procedures performed in the study involving human participants were in accordance with the ethical standards of the guidelines of the Declaration of Helsinki. The Chinese Longitudinal Healthy Longevity Survey (CLHLS) study was approved by research ethics committees of Duke University and Peking University (IRB00001052-13074). All participants provided written informed consent.

\section{Data and subjects}

Data used in this study were extracted from the latest Wave (2014) of the CLHLS. The CLHLS is an ongoing national population-based survey done in a randomly selected from half of the counties and cities in 22 of the 31 provinces, covering approximately $82 \%$ of the total population of People's Republic of China. ${ }^{18}$ A more 
detailed sampling procedure and quality of the CLHLS is reported elsewhere ${ }^{18}$ and specified document are available via the CLHLS website (http://centerforaging.duke.edu/chi nese-longitudinalhealthy-longevity-survey).

In the present study, participants who were over 60 years old and provided complete data on sociodemographic, physical functioning, Modified Mini-Mental State Examination (mMMSE), behavior items, and negative emotion items were included. Since responses who had low cognitive functioning were unlikely to complete the survey accurately and may further provide inaccurate information on their mental well-being and physical function, all observations with missing or "unable to answer" values were excluded from the analysis. This resulted in an analytic sample of 2570 OAs from 7192 subjects. Similar data processing method can be found in previews studies. ${ }^{1,19}$

\section{Measures}

Participants covered sociodemographic and four main areas of functioning, including 1a) physical functioning, 2) cognitive functioning, 3) behaviors, and 4) negative emotion.

- Sociodemographic factors: gender $\quad(1=$ male, $2=$ female), age and education (in years), category of residence $(1=$ city, $2=$ town, $3=$ rural), co-residence $(1=$ with household members, $2=$ alone, $3=$ in a situation), marital status ( $1=$ married and living with spouse, $2=$ married but not living with spouse, 3 =divorced, 4=widowed, 5=never married), selfrated economic status ( $1=$ very rich, $2=$ rich, $3=$ so so, $4=$ poor, $5=$ very poor).

1) Physical functioning: Physical functioning was indicated by the self-rated health, quality of sleep, activities of daily living $(\mathrm{ADL})^{20}$ measures, and the instrumental activities of daily living (IADL) ${ }^{21}$ measures. The respondents were required to rate their overall health as " $5=$ very good", "4=good", "3=so so","2=bad", or "1=very bad". Quality of sleep was measured with an item asking participants to evaluate their current sleep status ("5=very good", "4=good", "3=so so"," $2=$ bad", or " $1=$ very bad"). The items assessing ADL included basic activity of daily life, which were widely used to describe the physical functioning and disability of OAs. The following information was investigated from all participants, including whether they need others help to complete the following daily tasks: bathing, dressing, going to the toilet, indoor transferring, incontinence, and eating (coded with $3=$ need no assistance, $2=$ need assistance partly, and $1=$ need assistance fully). As for IADL, the respondents were asked whether they could complete eight tasks by themselves. The items of IADL included visiting neighbors, go shopping, cooking a meal, washing clothes, walking continuously for 1 $\mathrm{km}$ at a time, lifting a weight of $5 \mathrm{~kg}$, crouching and standing up continuously three times, and taking public transportation (coded with $3=$ fully independently, $2=$ need some help, and 1=no, cannot). The higher ADL and IADL scores indicated better physical functioning. Previous study has found that the Cronbach's alphas for ADL and IADL measures ranged from 0.86 to 0.99 at different waves, indicating good internal consistency. ${ }^{18}$

2) Cognitive functioning: The cognitive function of participants was assessed with a Chinese version of the mMMSE, ${ }^{2,23}$ including seven aspects of functioning: orientation to time, orientation to place, immediate registration, attention and calculation, delayed recall, language, and complex command. Each item of the mMMSE was recorded as 1 if the answer was correct and 0 for wrong. ${ }^{24}$ The higher scores suggested better cognitive function. The reliability of the mMMSE was high (Cronbach's $\alpha=0.98)^{24}$

3) Behaviors: Behavioral factors were measured by currently smoking $(1=$ yes, $0=$ no), currently consuming alcohol $(1=$ yes, $0=$ no), regularly exercising $(1=y e s$, $0=$ no), regularly physical labor $(1=$ yes, $0=$ no $)$, and frequency of social participation $(5=$ almost every day, $4=$ at least once a week, $3=$ at least once a month, $2=$ sometimes, $1=$ never).

4) Mental health: In the CLHLS, four items were used to create an index of emotional well-being, including two tap negative effects (anxiety and self-perceived uselessness) and two capture positive effects (happiness and optimism). ${ }^{25}$ We rated these items in a 5-point response format ("5=always", "4=often", " $3=$ sometimes", " $2=$ seldom", or " $1=$ never"). Afterward, the two positive items were coded reversely, followed by addition to the two negative items to compute the total score. Previous study reported that the reliability of negative emotion scale ranges from 0.58 to 0.65 for the CLHLS. ${ }^{6}$

\section{Data analysis}

Demographic characteristics and scores of scales were calculated. Continuous variables are expressed as mean and standard deviation. Categorical variables are expressed as percentages. The associations between 
variables were first examined using Pearson's correlation. Subsequently, multiple linear regression was used to assess the predictive effect, with negative emotion as the outcome variable and physical functioning, cognitive functioning, behaviors as predictors, followed with demographic characteristics. In addition, domain-specific models were used (health variables as predictors only), followed by enrollment of all predictors from those domain-specific models with predictive value $(P<0.05)$ in a combined model.

SPSS software version 21.0 was used for statistical analysis. A two-sided $P<0.05$ was considered as statistically significant.

\section{Results}

\section{Descriptive characteristics}

Of the 2570 observations, 1562 (60.78\%) were males, with a mean age of 80.20 years (SD: 8.60, range: 60-112). Detailed demographic characteristics of the sample are summarized in Table 1. The average year of education was 3.81 (SD: 3.86, ranging from 0 to 20 years). Almost half of the observations' residence were rural, followed by town. The most common co-residence was living with household members. Most of the participants were married and living with spouse, followed with widowed. In addition, $70 \%$ of the subjects rated their economic status as so so. Finally, the mean total scores of ADL, IADL, mMMSE, and negative emotion were $17.78(\mathrm{SD}=0.89), 21.93(\mathrm{SD}=3.69)$, $21.63(\mathrm{SD}=2.23)$, and $8.89(\mathrm{SD}=2.64)$, respectively.

\section{Correlations among central key study variables}

As indicated in Table 2, the results show that negative emotion was negatively associated with self-reported health ( $\mathrm{r}=-0.391, P<0.001)$, quality of sleep $(\mathrm{r}=-0.236$, $P<0.001)$, ADL $(\mathrm{r}=-0.100, P<0.001)$, IADL $(\mathrm{r}=-0.179$, $P<0.001)$, cognitive function ( $\mathrm{r}=-0.098, P<0.001)$, currently consuming alcohol $(\mathrm{r}=-0.053, P=0.007)$, regularly exercising $(\mathrm{r}=-0.208, P<0.001)$, and frequency of social participation $(\mathrm{r}=-0.123, P<0.001)$. In contrast, negative emotion was positively associated with regular physical labor $(\mathrm{r}=0.061, P=0.002)$. However, there are no obvious relationships between currently smoking $(\mathrm{r}=-0.003$, $P=0.879$ ) and negative emotion.

\section{Predicting negative emotion score}

The regression results for mental health are demonstrated in Table 3. The domain-specific models indicate that
Table I Demographic characteristics of sample

\begin{tabular}{|c|c|c|}
\hline \multirow[t]{2}{*}{ Characteristic } & \multicolumn{2}{|c|}{ Overall sample $(\mathrm{N}=\mathbf{2 5 7 0})$} \\
\hline & $\mathbf{N}$ & $\%$ \\
\hline Male & 1562 & 60.78 \\
\hline Age $(M \pm S D)$ & $80.20 \pm 8.60$ & \\
\hline Years of schooling $(M \pm S D)$ & $3.81 \pm 3.86$ & \\
\hline$A D L$ total score $(M \pm S D)$ & $17.78 \pm 0.89$ & \\
\hline IADL total score $(M \pm S D)$ & $21.93 \pm 3.69$ & \\
\hline MMSE total score $(M \pm S D)$ & $21.63 \pm 2.23$ & \\
\hline Negative emotion $(M \pm S D)$ & $8.89 \pm 2.64$ & \\
\hline \multicolumn{3}{|l|}{ Category of residence } \\
\hline City & 461 & 17.94 \\
\hline Town & 915 & 35.60 \\
\hline Rural & 1194 & 46.46 \\
\hline \multicolumn{3}{|l|}{ Co-residence } \\
\hline With household member(s) & 2055 & 79.96 \\
\hline Alone & 460 & 17.90 \\
\hline In an institution & 55 & 2.14 \\
\hline \multicolumn{3}{|l|}{ Marital status } \\
\hline Married and living with spouse & 1399 & 54.44 \\
\hline Married but not living with spouse & 49 & 1.91 \\
\hline Divorced & 13 & 0.51 \\
\hline Widowed & 1080 & 42.02 \\
\hline Never married & 29 & 1.13 \\
\hline \multicolumn{3}{|l|}{ Self-rated economic status } \\
\hline Very rich & 48 & 1.87 \\
\hline Rich & 482 & 18.75 \\
\hline So so & 1818 & 70.74 \\
\hline Poor & 190 & 7.39 \\
\hline Very poor & 32 & 1.25 \\
\hline
\end{tabular}

Abbreviations: ADL, activities of daily living; IADL, instrumental activities of daily living; mMMSE, Modified Mini-Mental State Examination.

overall demographic model, physical functioning model, cognitive functioning model, and behaviors model were all significantly predicted mental health $(P<0.001)$. In combined model, negative emotion was associated with being female, living in rural, self-perceived low economic status, poor self-rated health condition, poor sleep quality, low IADL function, and less regular exercising.

\section{Discussion}

A comprehensive understanding of the detrimental effect of physical, behavior, and cognitive, particularly the distinct effects of multiple aspects, on mental health could shed light on the design of psychosocial and 


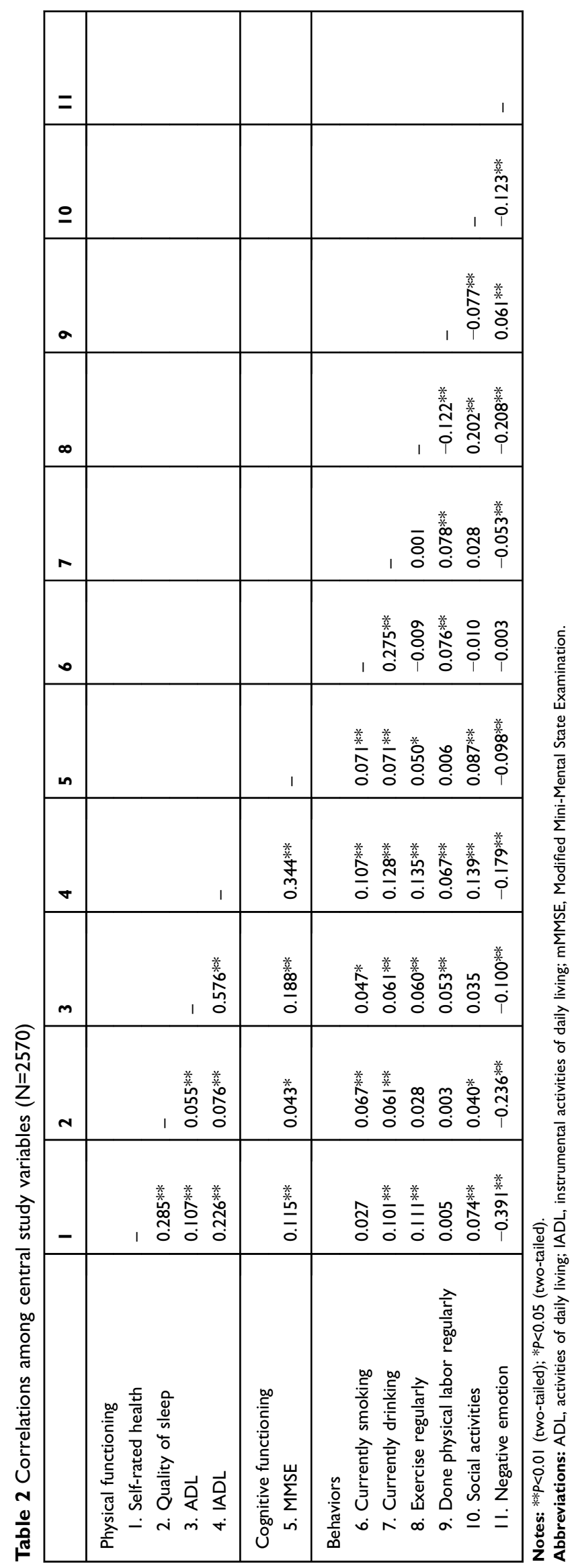


Table 3 Regression models predicting negative emotion: domain-Specific and combined models ( $N=2570)$

\begin{tabular}{|c|c|c|c|c|}
\hline Variable & B & SE & $\boldsymbol{\beta}$ & $P$-value \\
\hline \multicolumn{5}{|c|}{ Demographic model (final model adjusted $R^{2}=0.095, F(7,2562)=39.48 I, P<0.00 I$ ) } \\
\hline Gender & 0.457 & 0.113 & 0.084 & $<0.001$ \\
\hline Age at testing & 0.003 & 0.006 & 0.011 & 0.596 \\
\hline Years of schooling & -0.036 & 0.014 & -0.052 & 0.014 \\
\hline Category of residence & 0.440 & 0.069 & 0.125 & $<0.001$ \\
\hline Co-residence & 0.164 & 0.120 & 0.029 & 0.172 \\
\hline Marital status & 0.059 & 0.042 & 0.033 & 0.161 \\
\hline Self-rated economic status & -0.969 & 0.083 & -0.223 & $<0.001$ \\
\hline \multicolumn{5}{|c|}{ Physical functioning model (final model adjusted $R^{2}=0.177, F(4,2565)=|39.096, P<0.00|$ ) } \\
\hline Self-rated health & -1.014 & 0.058 & -0.332 & $<0.001$ \\
\hline Quality of sleep & -0.389 & 0.054 & -0.134 & $<0.001$ \\
\hline$A D L$ & -0.012 & 0.065 & -0.004 & 0.849 \\
\hline IADL & -0.065 & 0.016 & -0.091 & $<0.001$ \\
\hline \multicolumn{5}{|c|}{ Cognitive functioning model (final model adjusted $R^{2}=0.009, F(I, 2568)=24.725, P<0.00 I$ ) } \\
\hline MMSE & -0.116 & 0.023 & -0.098 & $<0.001$ \\
\hline \multicolumn{5}{|c|}{ Behaviors model (final model adjusted $R^{2}=0.052, F(5,2564)=29.263, P<0.00 I$ ) } \\
\hline Currently smoking ( $I=y e s, 0=$ no) & 0.043 & 0.125 & 0.007 & 0.733 \\
\hline Currently drinking $(I=y e s, 0=$ no) & -0.351 & 0.128 & -0.055 & 0.006 \\
\hline Exercise regularly $(I=y e s, 0=$ no $)$ & -1.001 & 0.106 & -0.187 & $<0.001$ \\
\hline Done physical labor regularly $(I=y e s, 0=$ no $)$ & 0.236 & 0.130 & 0.035 & 0.069 \\
\hline Social activities $(5=$ almost everyday, $I=$ never $)$ & -0.203 & 0.049 & -0.081 & $<0.001$ \\
\hline \multicolumn{5}{|c|}{ Combined model (final model adjusted $R^{2}=0.237, F(I I, 2558)=73.583, P<0.00 I$ ) } \\
\hline Gender & 0.338 & 0.105 & 0.063 & 0.001 \\
\hline Years of schooling & -0.021 & 0.013 & -0.031 & 0.111 \\
\hline Category of residence & 0.370 & 0.065 & 0.105 & $<0.001$ \\
\hline Self-rated economic status & -0.572 & 0.078 & -0.132 & $<0.001$ \\
\hline Self-rated health & -0.890 & 0.058 & -0.291 & $<0.001$ \\
\hline Quality of sleep & -0.332 & 0.053 & -0.114 & $<0.001$ \\
\hline IADL & -0.057 & 0.014 & -0.080 & $<0.001$ \\
\hline MMSE & 0.001 & 0.022 & 0.001 & 0.971 \\
\hline Currently drinking $(I=y e s, 0=$ no $)$ & 0.068 & 0.116 & 0.011 & 0.555 \\
\hline Exercise regularly $(\mathrm{I}=\mathrm{yes}, 0=\mathrm{no})$ & -0.550 & 0.099 & -0.103 & $<0.001$ \\
\hline Social activities ( $5=$ almost everyday, $I=$ never $)$ & -0.078 & 0.045 & -0.031 & 0.081 \\
\hline
\end{tabular}

Note: Variable selection for combined models was based on including all variables from domain-specific models with $P \leq 0.05$.

Abbreviations: ADL, activities of daily living; IADL, instrumental activities of daily living; mMMSE, Modified Mini-Mental State Examination.

environmental interventions to prevent negative emotion. In this study, the 2014 CLHLS was utilized to investigate the relationship between demographic, physical functioning, behavior, cognitive, and mental health. The results revealed that the negative effect of physical, behavior, and cognitive played a significant role on negative emotion among Chinese OAs.

Similar to previous studies, ${ }^{26,27}$ our results showed that females were more likely to susceptible to negative emotion, which might be related to the fact that women, instead of men, are sensitive to emotionally negative stimuli of lesser saliency. $^{26}$ We also find that education is related to mental health. Similarly, in a study from Korea, ${ }^{28}$ which reported that education was significantly correlated with self-reported mental health literacy, in other words, subjects with more education were more likely to have higher levels of mental health literacy. However, to our surprise, participants living in rural area and lower self-rated economic status were more likely to 
have higher levels negative emotion, possibly due to the limited social resources and support in rural residence, which may restrict the way to let negative emotion outpour in OAs. Our findings have important policy implications that information and education programs on psychological well-being could be included in the program of rural area community service among OAs.

Another important finding is that OAs with higher level physical function reported low level of negative emotion. It is feasible that those physiologically stronger individuals are able to live with chronic disease, and the survival advantage may be possibly attributed to positive psychological function. This finding is in line with the results from Jopp, ${ }^{16}$ who reported subjective physical function was a significant negative predictor for depression, and IADL was positively significant for life satisfaction. Kunzmann ${ }^{29}$ also noted that, subjective health was the strongest predictor for mental health in all regression, replicating studies among younger populations' models. In turn, emotion regular strategies play an important role in physical function. ${ }^{30,31}$ However, there is also inconsistent research finding, revealing that centenarians reported high levels of well-being were found to be as happy as middle-aged adults, despite of health limitations. ${ }^{32}$ Still, longitudinal data are required to verify the association between physical function and mental health and to estimate the effect size of mental intervention programs.

Our present findings suggest that participants with drinking, regular exercising, and frequent social activity participation are more likely to have low negative emotion. Consistently, previous studies ${ }^{33,34}$ have also reported that drinking is perceived to play an important role in relieving fatigue, easing tension and facilitating social exchanges in social gatherings. Alcohol consumption has a long history in Chinese culture among men. In general, compared with women, men have more social interactions to drink. It is reported that in rural areas of People's Republic of China, men may drink to relieve physical fatigue since are more likely to do the heavy labor than women. ${ }^{35}$ This view is also applicable to smoking. To be specific, these findings may explain Wang's $^{36}$ study, which revealed that women were less likely to be current smokers and frequent drinkers, but had higher prevalence of poor mental health compared with their male counterparts. Further research is warranted to explore the impact of smoking or drinking on the mental health based on overall dietary patterns and multiple $24 \mathrm{hrs}$ food records, especially among the very selective oldest-old population.
Practically, the present study provides further evidence that OAs with better cognitive function were more likely to have a positive mental condition, while those under negative mental status may have cognitive deficits. Previous studies have indicated that mental health could significantly predict age-related neurological disorders, including mild cognitive impairment, ${ }^{37}$ mild dementia, ${ }^{38}$ and Parkinson's disease. ${ }^{39}$ Moreover, our study extends this finding to detailed mental health constructs, such as anxiety and self-perceived useless. Clearly, further research is required to confirm this clinical potential.

The strength of this study was the use of national-level data based on face-to-face interviews. Our findings fill in gaps in the research about a comprehensive picture of central areas of functioning among OAs living in Asian societies.

However, several limitations of this study must also be noted. Firstly, all measurements were based on self-report which can introduce selection bias, such as inherent misreporting, social desirability, and recall error. Second, no more detailed information of the frequency of drinking, type of alcohol beverage, amount of alcohol intake, or alcohol-related disorders available from the datasets, therefore we were unable to conduct a deeper insightful investigation about the relationships between alcohol consumption, smoking, and other reported behaviors. A more precise definition of type, frequency, quantity of drinking, and smoking is warranted in future studies. Third, it is also possible that cognitive deterioration could lead to negative emotion by causing impairments in individuals' ability to maintain psychological well-being, communicate with others, and participation in social activities. However, it is generally unlikely to explore this possibility using the present analytic sample, since its simplicity that gives a rough approach to OAs' cognitive function, which warrants some degree of caution. Finally, although the scale of negative emotion was previously used in these datasets, not all mental variables were tested. Therefore, a more precise definition of negative emotion and reliable means should be developed and validated in future studies.

\section{Conclusion}

In People's Republic of China, the one-child policy and internal migration from rural areas to urban centers by workers have resulted in large increases "empty-nested elders". ${ }^{40}$ Among them, almost 80\% reported high levels loneliness and other negative emotion, which may exacerbate the problem of dementia and cognitive decline in 
People's Republic of China. ${ }^{40,41}$ Hence, from the perspective of public health, the disease burden of cognitive decline in People's Republic of China could be reduced through effective interventions, aiming at releasing negative emotion, especially among the population with chronic loneliness. Future studies should develop a more comprehensive and multidimensional approach to obtain a better understanding of the resilience mechanisms and potential risks for negative emotion, so as to ensure a better quality of life for the increasing number of OAs in remaining years, even a small reduction or delay in the progression of cognitive impairment. Besides extending our understanding, our findings would also help to provide guidance for healthy behaviors as well as develop support structure and services for living in the community.

\section{Abbreviations}

OAs, old adults; CLHLS, Chinese Longitudinal Healthy Longevity Survey; mMMSE, Modified Mini-Mental State Examination; ADL, activities of daily living; IADL, instrumental activities of daily living.

\section{Acknowledgment}

We gratefully acknowledge Duke University and Peking University for giving us the permission to use the data of the Chinese Longitudinal Healthy Longevity Survey conducted in 2014.

\section{Author contributions}

All authors contributed to data analysis, drafting or revising the article, gave final approval of the version to be published, and agree to be accountable for all aspects of the work.

\section{Disclosure}

The authors report no conflicts of interest in this work.

\section{References}

1. Zhang Y, Gu Y, Zhang Y, et al. Effect of sociodemographic and physical activity on cognitive function in older adults: A nationwide cross-sectional survey. Int J Geriatr Psych. 2018;34(2):243-248. doi:10.1002/gps.4932

2. Wu Y, Dang J. Blue Book of Aging: China Report of the Development on Aging Cause. Beijing: Social Sciences Academic Press (China); 2013.

3. Blaxland M, Shang X, Fisher KR. Introduction: people oriented: A new stage of social welfare development in China. J Soc Serv Res. 2014;40(4):508-519. doi:10.1080/01488376.2014.923801

4. Ezeh AC, John B, Blessing M. Global population trends and policy options. Lancet. 2012;380(9837):142-148. doi:10.1016/S0140-6736 (12)60696-5
5. KW BOEY. Aging and mental health problems: A study on the elderly in urban China. Aging Ment Health. 1997;1(4):332-338. doi:10.1080/13607869757029

6. Zhong BL, Chen SL, Conwell Y. Effects of transient versus chronic loneliness on cognitive function in older adults: findings from the Chinese longitudinal healthy longevity survey. Am $J$ Geriatr Psychiatry. 2016;24(5):389-398. doi:10.1016/j.jagp.2015.12.009

7. Zhang W, Ding H, Su P, et al. Does disability predict attempted suicide in the elderly? A community-based study of elderly residents in Shanghai, China. Aging Ment Health. 2016;20(1):81-87. doi:10.1080/13607863.2015.1031641

8. Zhong BL, Chiu HFK, Conwell Y. Rates and characteristics of elderly suicide in China, 2013-14. J Affect Disorders. 2016;206:273-279. doi:10.1016/j.jad.2016.09.003

9. Park JI, Yang JC, Han C, Park TW, Chung SK. Suicidal ideation among Korean elderly: risk factors and population attributable fractions. Psychiatry-interpersonal Biol Processes. 2016;79(3):262-281. doi:10.1080/00332747.2016.1175837

10. Kim HS, Bae NK, Kwon IS, Cho YC. Relationship between status of physical and mental function and quality of life among the elderly people admitted from long-term care insurance. J Prev Med Public Health. 2010;43(4):319. doi:10.3961/jpmph.2010.43.4.319

11. Moon JJ, Lee BJ, Kim JE, et al. A correlation of cognitive function, depression, suicidal idea and serum lipid levels in the elderly over sixty years of age. Korean J Biol Psychiatry. 2010;17:210.

12. North MS, Fiske ST. Modern attitudes toward older adults in the aging world: a cross-cultural meta-analysis. Psychol Bull. 2015;141 (5):993-1021. doi:10.1037/a0039469

13. Zhao Y, Sautter JM, Qiu L, Gu D. Self-perceived uselessness and associated factors among older adults in China. BMC Geriatr. 2017;17(1):12. doi:10.1186/s12877-017-0515-3

14. Shakoor H, Andy H, Christopher B, Astrid F. Patterns and determinants of alcohol consumption in people aged 75 years and older: results from the MRC trial of assessment and management of older people in the community. Age Ageing. 2004;128(1-2):170-177.

15. Li J, Bei W, Selbæk G, Krokstad S, Helvik AS. Factors associated with consumption of alcohol in older adults - a comparison between two cultures, China and Norway: the CLHLS and the HUNT-study. BMC Geriatr. 2017;17(1):172. doi:10.1186/s12877-017-0515-3

16. Jopp DS, Park MKS, Lehrfeld J, Paggi ME. Physical, cognitive, social and mental health in near-centenarians and centenarians living in New York City: findings from the Fordham Centenarian Study. BMC Geriatr. 2016;16(1):1. doi:10.1186/s12877-015-0167-0

17. Puvill T, Lindenberg J, Craen AJMD, Slaets JPJ, Westendorp RGJ. Impact of physical and mental health on life satisfaction in old age: a population based observational study. BMC Geriatr. 2016;16(1):194. doi:10.1186/s12877-016-0365-4

18. Goodkind D. Healthy longevity in China: demographic, socioeconomic, and psychological dimensions. 2008 by ZENG YI; DUDLEY L. POSTON; DENESE ASHBAUGH VLOSKY; DANAN GU. Popul Stud (Camb). 2009;63(3):312-313.

19. Zhenmei Z, Danan G, Hayward MD. Childhood nutritional deprivation and cognitive impairment among older Chinese people. Soc Sci Med. 2010;71(5):941-949. doi:10.1016/j.socscimed.2010.05.013

20. Applegate WB, Blass JP, Williams TF. Instruments for the functional assessment of older patients. N Engl J Med. 1990;322(17):12071214. doi:10.1056/NEJM199004263221707

21. Lawton MP, Brody EM. Assessment of Older people: self-maintaining and instrumental activities of daily living. Gerontologist. 1969;9 (3 Part 1):179-186.

22. Folstein MF, Folstein SE, Mchugh PR. "Mini-mental state": A practical method for grading the cognitive state of patients for the clinician. J Psychiatr Res. 1975;12(3):189-198.

23. Francesco L, Zhen Z. A missing composite covariate in survival analysis: a case study of the Chinese Longitudinal Health and Longevity Survey. Stat Med. 2010;29(2):248-261. doi:10.1002/sim.3773 
24. Zhang Z, Gu DM. Early life influences on cognitive impairment among oldest old Chinese. $J$ Gerontol. 2008;63(1):S25. doi:10.1093/geronb/63.1.S25

25. Luo Y, Zhang Z, Gu D. Education and mortality among older adults in China. Soc Sci Med. 2014;69(4):134-142.

26. Hong L, Jiajin Y, Chongde L. The neural mechanism underlying the female advantage in identifying negative emotions: an event-related potential study. Neuroimage. 2008;40(4):1921-1929. doi:10.1016/j. neuroimage.2008.01.033

27. Yuan J, Luo Y, Yan JH, Meng X, Yu F, Li H. Neural correlates of the females' susceptibility to negative emotions: an insight into genderrelated prevalence of affective disturbances. Hum Brain Mapp. 2010;30(11):3676-3686. doi:10.1002/hbm.20796

28. Kim YS, Lee HY, Lee MH, Simms T, Park BH. Mental health literacy in Korean older adults: a cross-sectional survey. J Psychiatr Mental Health Nurs. 2017;24(7). doi:10.1111/jpm.12395

29. Kunzmann U. Sources of well-being in very old age. Brittonia. 1999;16(1):54-57.

30. Talaei-Khoei M, Nemati-Rezvani H, Fischerauer SF, Ring D, Chen $\mathrm{N}$, Vranceanu AM. Emotion regulation strategies mediate the associations of positive and negative affect to upper extremity physical function. Compr Psychiat. 2017;75:85. doi:10.1016/j.comppsych. 2017.03.005

31. Gallo LC, Matthews KA. Understanding the association between socioeconomic status and physical health: do negative emotions play a role? Psychol Bull. 2003;129(1):10-51.

32. Wong WCP, Lau HPB, Kwok CFN, et al. The well-being of communitydwelling near-centenarians and centenarians in Hong Kong a qualitative study. BMC Geriatr. 2014;14(1):63. doi:10.1186/1471-2318-14-63

33. Lin TY, Lin DTC. Alcoholism among the chinese: further observations of a low-risk population. Cult Med Psychiatry. 1982;6(2):109116. doi:10.1007/BF00051426
34. Wei H. Alcohol policy and the public good: a Chinese view. Addiction. 2010;90(11):1448-1450. doi:10.1111/j.1360-0443.1995. tb02803.x

35. Zhou X, Su Z, Deng H, Xiang X, Chen H, Hao W. A comparative survey on alcohol and tobacco use in urban and rural populations in the Huaihua District of Hunan Province, China. Alcohol. 2006;39 (2):87-96. doi:10.1016/j.alcohol.2006.07.003

36. Wang S, Ungvari GS, Forester BP, et al. Gender differences in general mental health, smoking, drinking and chronic diseases in older adults in Jilin province, China. Psychiat Res. 2017;251:58. doi:10.1016/j.psychres.2017.02.007

37. Bielak AA, Hultsch DF, Strauss E, Macdonald SW, Hunter MA. Intraindividual variability in reaction time predicts cognitive outcomes 5 years later. Neuropsychology. 2010;24(6):731. doi:10.1037/ a0019312

38. Dixon RA, Garrett DD. Neurocognitive markers of cognitive impairment: exploring the roles of speed and inconsistency. Neuropsychology. 2007;3(21):381-399. doi:10.1037/0894-4105.21.3.381

39. De-Frias C, Dixon R, Fisher N, Camicioli R. Intraindividual variability in neurocognitive speed: a comparison of Parkinson's disease and normal older adults. Neuropsychologia. 2007;45(11):2499-2507. doi:10.1016/j.neuropsychologia.2007.03.022

40. Chen Y, Hicks A, While AE. Loneliness and social support of older people in China: a systematic literature review. Health Soc Care Comm. 2014;22(2):113-123. doi:10.1111/hsc.12051

41. Zhen-Qiang W, Liang S, Ye-Huan S, Xiu-Jun Z, Fang-Biao T, Guang-Hui C. Correlation between loneliness and social relationship among empty nest elderly in Anhui rural area, China. Aging Ment Health. 2010;14(1):108-112. doi:10.1080/13607860903228796
Psychology Research and Behavior Management

\section{Publish your work in this journal}

Psychology Research and Behavior Management is an international, peer-reviewed, open access journal focusing on the science of psychology and its application in behavior management to develop improved outcomes in the clinical, educational, sports and business arenas. Specific topics covered in the journal include: Neuroscience, memory and decision making; Behavior modification and management; Clinica applications; Business and sports performance management; Social and developmental studies; Animal studies. The manuscript management system is completely online and includes a very quick and fair peer-review system, which is all easy to use. Visit http://www. dovepress.com/testimonials.php to read real quotes from published authors. 